\title{
PICASSO, COUPP and PICO - search for dark matter with bubble chambers
}

C. Amole ${ }^{1}$, M. Ardid ${ }^{2}$, D. M. Asner ${ }^{3}$, D. Baxter 4 , E. Behnke ${ }^{5}$, P. Bhattacharjee ${ }^{6}$, H. Borsodi ${ }^{5}$, M. Bou-Cabo $^{2}$, S. J. Brice ${ }^{7}$, D. Broemmelsiek ${ }^{7}$, K. Clark ${ }^{8}$, J. I. Collar ${ }^{9}$, P. S. Cooper ${ }^{7}$, M. Crisler ${ }^{7}$, C. E. Dahl ${ }^{4}$, M. Das ${ }^{6}$, F. Debris ${ }^{10}$, N. Dhungana ${ }^{11}$, J. Farine ${ }^{11}$, I. Felis ${ }^{2}$, R. Filgas ${ }^{12, a}$, M. FinesNeuschild $^{10}$, F. Girard ${ }^{10}$, G. Giroux ${ }^{1}$, M. Hai ${ }^{9}$, J. Hall ${ }^{3}$, O. Harris ${ }^{5}$, C. M. Jackson ${ }^{10}$, M. Jin ${ }^{4}$, C. Krauss $^{13}$, M. Lafrenière ${ }^{10}$, M. Laurin ${ }^{10}$, I. Lawson ${ }^{14,11}$, I. Levine ${ }^{5}$, W. H. Lippincott ${ }^{7}$, E. Mann ${ }^{5}$, J. P. Martin ${ }^{10}$, D. Maurya ${ }^{15}$, P. Mitra ${ }^{13}$, R. Neilson ${ }^{9,16}$, A. J. Noble ${ }^{1}$, A. Plante ${ }^{10}$, R. Podviyanuk ${ }^{11}$, S. Priya ${ }^{15}$, A. E. Robinson ${ }^{9}$, M. Ruschman ${ }^{7}$, O. Scallon ${ }^{11,10}$, S. Seth ${ }^{6}$, A. Sonnenschein ${ }^{7}$, N. Starinski ${ }^{10}$, I. Štekl ${ }^{12}$, E. Vázquez-Jáuregui ${ }^{14,11}$, J. Wells ${ }^{5}$, U. Wichoski ${ }^{11}$, V. Zacek ${ }^{10}$, and J. Zhang $^{4}$ (PICO Collaboration)

${ }^{1}$ Department of Physics, Queen's University, Kingston, K7L 3N6, Canada

${ }^{2}$ Universitat Politècnica de València, IGIC, 46730 Gandia, Spain

${ }^{3}$ Pacific Northwest National Laboratory, Richland, Washington 99354, USA

${ }^{4}$ Department of Physics, Northwestern University, Evanston, Illinois 60208, USA

${ }^{5}$ Department of Physics, Indiana University South Bend, South Bend, Indiana 46634, USA

${ }^{6}$ Astroparticle Physics and Cosmology Division, Saha Institute of Nuclear Physics, Kolkata, 700064, India

${ }^{7}$ Fermi National Accelerator Laboratory, Batavia, Illinois 60510, USA

${ }^{8}$ Department of Physics, University of Toronto, Toronto, ON M5S 1A7, Canada

${ }^{9}$ Enrico Fermi Institute, KICP and Department of Physics, University of Chicago, Chicago, Illinois 60637, USA

${ }^{10}$ Département de Physique, Université de Montréal, Montréal, H3C 3J7, Canada

${ }^{11}$ Department of Physics, Laurentian University, Sudbury, P3E 2C6, Canada

${ }^{12}$ Institute of Experimental and Applied Physics, Czech Technical University in Prague, Prague, 12800, Czech Republic

${ }^{13}$ Department of Physics, University of Alberta, Edmonton, T6G 2G7, Canada

${ }^{14}$ SNOLAB, Lively, Ontario, P3Y 1N2, Canada

${ }^{15}$ Center for Intelligent Material Systems and Structures, Virginia Tech, Blacksburg, Virginia 24061, USA

${ }^{16}$ Department of Physics, Drexel University, Philadelphia, Pennsylvania 19104, USA

\begin{abstract}
The PICASSO and COUPP collaborations use superheated liquid detectors to search for cold dark matter through the direct detection of weakly interacting massive particles (WIMPs). These experiments, located in the underground laboratory of SNOLAB, Canada, detect phase transitions triggered by nuclear recoils in the $\mathrm{keV}$ range induced by interactions with WIMPs. We present details of the construction and operation of these detectors as well as the results, obtained by several years of observations. We also introduce PICO, a joint effort of the two collaborations to build a second generation ton-scale bubble chamber with 250 liters of active liquid.
\end{abstract}

\footnotetext{
ae-mail: robert.filgas@utef.cvut.cz
} 


\section{Introduction}

One of the key scientific questions of the cosmology and particle physics today involves unveiling the elusive nature of dark matter. In the last thirty years, gravitational evidence for dark matter has been accumulated on the scales ranging from galactic to cosmological. While these gravitational evidences are conclusive as to the existence of dark matter, they do not fully shed light on its microscopic properties. The consistency with the current cosmology model and the observational evidence of the lack of baryonic mass point toward collisionless and dissipationless non-baryonic dark matter. Moreover, the focus is presently on non-relativistic (cold) dark matter candidates. Summarizing all the above conditions, the hypothetical candidate for dark matter has been called the Weakly Interacting Massive Particle (WIMP). Of the many WIMP candidates, probably the best motivated and certainly the most theoretically developed is the neutralino, the lightest superpartner in many supersymmetric theories [1]. There is now a vast experimental effort being conducted to detect WIMPs, an extremely challenging task given that they interact only weakly and gravitationally.

Direct detection experiments search for the WIMP scattering off atomic nuclei within the detectors, which are typically located in deep underground laboratories to reduce the cosmic rays background. The majority of these experiments are searching for dark matter by either detecting the heat produced when WIMP hits an atom in a crystal absorber such as germanium or by detecting the flash of a scintillation light produced by WIMP collision in liquefied noble gases. There is, however, a relatively new group of detectors attempting to detect WIMPs using superheated liquids, a technique inspired by the bubble chamber [2], [3]. These detectors search for for cold dark matter through the direct detection of neutralinos via their spin-dependent interactions with ${ }^{19} \mathrm{~F}$ nuclei since, with the exception of neutralino scattering on free protons, ${ }^{19} \mathrm{~F}$ is the most favorable nucleus for direct detection [4], [5]. In this paper, we present the details of our three experiments based on this technique and their search for dark matter.

\section{PICASSO}

The PICASSO experiment (Project in CAnada to Search for Super-symmetric Objects) specializes in searches for cold dark matter through the direct detection of WIMP scattering using superheated liquid droplets of perfluorobutane $\mathrm{C}_{4} \mathrm{~F}_{10}$. These droplets of about $200 \mu \mathrm{m}$ diameter are dispersed in a polymerized water saturated acrylamide. With a boiling temperature $\mathrm{T}_{b}=-1.7^{\circ} \mathrm{C}$ at a pressure of 1.013 bar this liquid is at ambient pressure and temperature in a moderately metastable superheated state. A heat spike created by the energy deposition of a charged particle traversing the liquid triggers a phase transition if the energy deposition occurs within a certain critical length (of the order of hundreds of $\mathrm{nm}$ ), and if the deposited energy exceeds a certain critical energy (in the keV range). Both of these quantities decrease exponentially with increasing temperature and are functions of surface tension, latent heat of evaporation and superheat, where the latter is defined as the difference between the vapor pressure of the liquid and the external pressure. Details of the detector principle are explained in [6], [7]. The phase transition from the liquid droplet to the vapour bubble is explosive in nature and is accompanied by an acoustic signal in the audible and ultrasonic region, which is recorded by piezoelectric transducers.

Given that this type of detector captures phase transitions, it performs as an energy threshold device which can be controlled by setting the temperature and/or pressure. The dependence of the threshold energy on the operating temperature has been determined by measurements using monoenergetic neutron beams (neutron induced recoils are similar to those of WIMPs) and with $\alpha$ emitters of known energies. The results of these calibrations are shown in a form of a calibration curve in Fig. 1 and allow a precise description of the temperature dependence of energy thresholds ranging from 

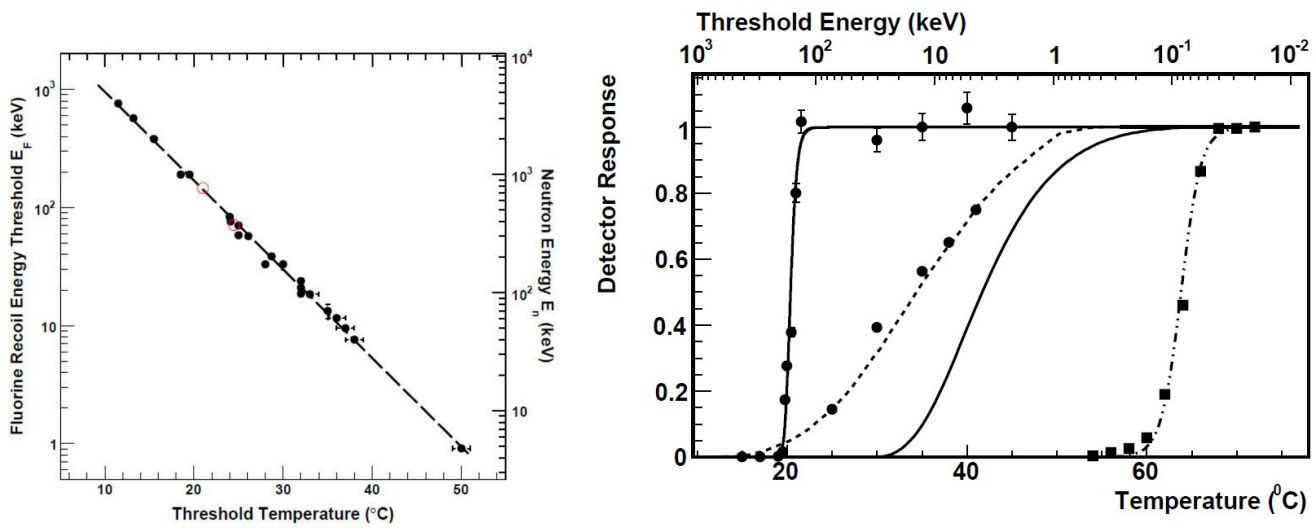

Figure 1: Left: Calibration curve for the energy threshold for ${ }^{19} \mathrm{~F}$ recoils as a function of temperature obtained from measurements with mono-energetic neutrons; $\alpha$-data are shown as open (red) circles. Right: Detector response to different types of particles as a function of temperature. From left to right: alpha particles of $5.6 \mathrm{MeV}$ in a detector spiked with ${ }^{226} \mathrm{Ra}$ (fit to data points represented by continuous line); nuclear recoils from fast neutrons of an AmBe source compared to simulations (dotted line); expected response for nuclear recoils following scattering of a $50 \mathrm{GeVc}^{-2}$ WIMP (continuous line); response to $1.275 \mathrm{MeV}$ gamma rays of a ${ }^{22} \mathrm{Na}$ source (dashed line). All responses are normalized to one at full detection efficiency. If not visible, experimental errors are smaller than the symbols. From [10]

$0.9 \mathrm{keV}$ up to $800 \mathrm{keV}$. Details of these calibration measurements can be found in [8], [9]. Since each temperature at a fixed constant pressure corresponds to a defined recoil energy threshold, the variation of the threshold temperature can be used to reconstruct the spectrum of the particle induced energy depositions. A measurement summary in Fig. 1 shows that the detector is insensitive to cosmic muons, $\gamma$ and $\beta$ radiation below $50{ }^{\circ} \mathrm{C}$. These minimum ionizing particles become detectable when they create sub-keV energy clusters within sub-nm sizes regions and are therefore well separated from strongly ionizing WIMP or neutron induced recoils, which allows efficient suppression of such backgrounds at the level of $10^{-8}$ to $10^{-10}$. WIMP induced recoil energies of ${ }^{19} \mathrm{~F}$ nuclei are expected to be smaller than $100 \mathrm{keV}$ and therefore become detectable above $30{ }^{\circ} \mathrm{C}$ (at 1 bar). However, Fig. 1 shows that the detector is fully sensitive to $\alpha$ particles over the entire range of the WIMP sensitivity. Therefore, $\alpha$ particles are the dominant source of the background for this type of the detector.

Before the PICASSO run ended in December 2013 and the detector was dismantled, the experiment consisted of 32 modules, installed in groups of four inside thermally and acoustically insulated temperature a pressure control units with a temperature range from $20^{\circ} \mathrm{C}$ to $50{ }^{\circ} \mathrm{C}$ and a precision of $\pm 0.1^{\circ} \mathrm{C}$. Each cylindrical module of $14 \mathrm{~cm}$ diameter and $40 \mathrm{~cm}$ of height [10] was fabricated from acrylic and filled with 4.5 litres of polymerized emulsion loaded with droplets of $\mathrm{C}_{4} \mathrm{~F}_{10}$. On average the active mass of a single module was $90 \mathrm{~g}$ of $\mathrm{C}_{4} \mathrm{~F}_{10}$ corresponding to $72 \mathrm{~g}$ of ${ }^{19} \mathrm{~F}$, yielding a total active mass of $2.6 \mathrm{~kg}$ of $\mathrm{C}_{4} \mathrm{~F}_{10}$. The active part of each detector is topped by mineral oil, which is connected to a hydraulic manifold in order to allow periodic pressurizations of the detectors to reconvert bubbles back into droplets. A WIMP run typically lasted 40 hours after which the detectors were recompressed for 15 hours at a pressure of 6 bar in order to reduce bubbles to droplets and to 

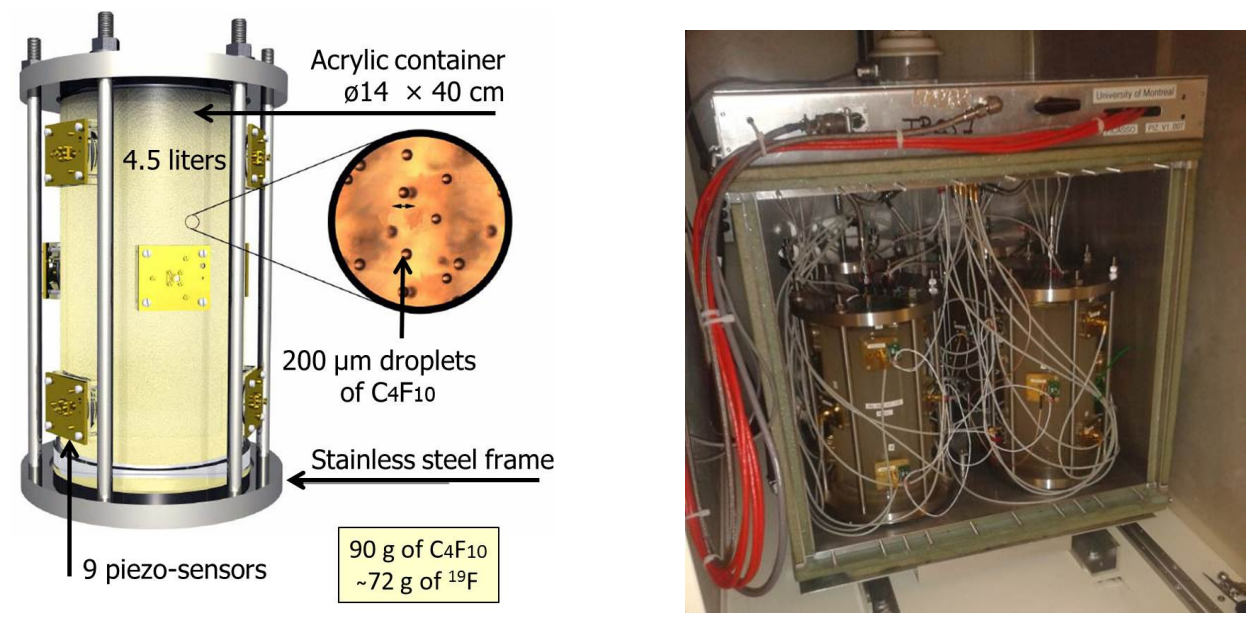

Figure 2: Left: Image of one of the 32 PICASSO modules showing the acrylic cylinder filled with gel matrix containing droplets of $\mathrm{C}_{4} \mathrm{~F}_{10}$. Right: One of the temperature and pressure control units housing 4 modules.

prevent excessive bubble growth which could damage the polymer. Each module is equipped with 9 piezoelectric transducers for the electronic readout.

To reduce the neutron background the whole experiment is surrounded by a $50 \mathrm{~cm}$ thick water shield and placed in the SNOLAB underground facility at a depth of $2070 \mathrm{~m}$. Mitigation of the alpha background was, however, a much more difficult as it cannot be shielded from outside. Possible $\alpha$ sources include a direct contamination of the $\mathrm{C}_{4} \mathrm{~F}_{10}$ itself or diffusion of ${ }^{222} \mathrm{Rn}$ from ${ }^{226} \mathrm{Ra}$ in the polymer matrix into the droplets Fortunately, PICASSO discovered a technique for an event by event $\alpha$ recoil discrimination using the acoustic signal energy. It was observed that the acoustic signals produced by $\alpha$-emitters are a factor four more intense than signals of neutron or WIMP induced events [11], see Fig. 3. This result has been confirmed by COUPP [12] and a theoretical model proposed by PICASSO is discussed in [9].

The abundance of ${ }^{19} \mathrm{~F}$ in the target liquid $\mathrm{C}_{4} \mathrm{~F}_{10}$ gives PICASSO an increased sensitivity to spin dependent WIMP interactions. The light target nucleus ${ }^{19} \mathrm{~F}$ together with the low recoil detection threshold of $1.7 \mathrm{keV}$ render the experiment particularly sensitive to low WIMP masses below 15 $\mathrm{GeV} / \mathrm{c}^{2}$. This is especially interesting following the DAMA/LIBRA and CoGeNT and CRESST results [14], [15], [16] which are suggestive of a low mass WIMP solution of order $10 \mathrm{GeV} / \mathrm{c}^{2}$. During its lifetime, PICASSO explored both the implications of the obtained data for searches in the spindependent sector, and the sensitivity to the low mass region in the spin-independent sector. Results of the WIMP search with PICASSO were published in 2009 [10] and 2012 [17] and a publication with the complete dataset is currently in preparation. 


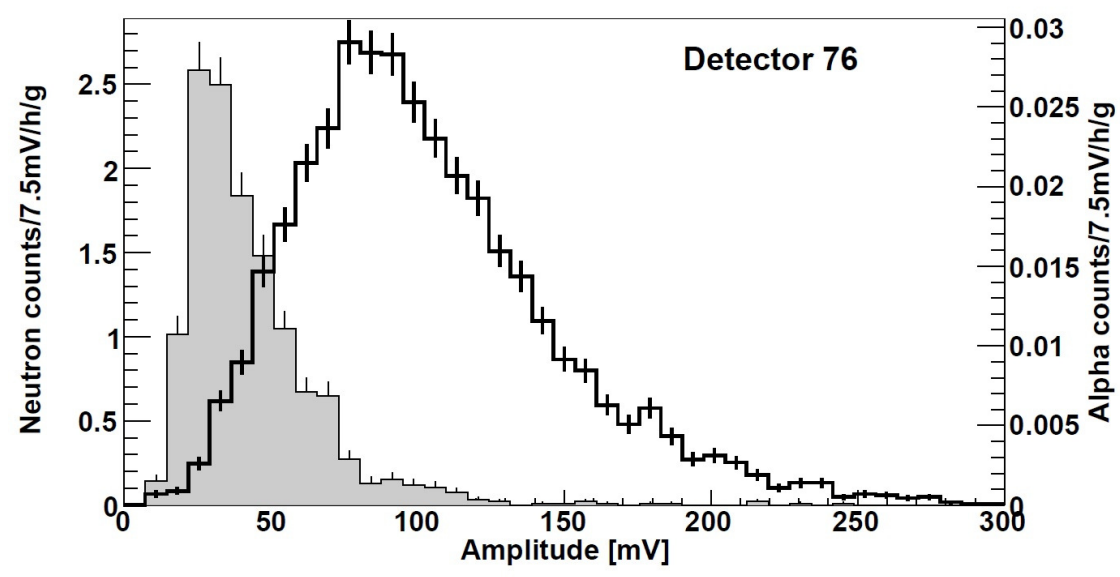

Figure 3: Amplitude distributions of neutron and alpha particle induced events for detectors 76 at 35 ${ }^{\circ} \mathrm{C}$. Plotted is the average of the peak amplitudes of nine transducers. Count rates are given per active mass. Neutron data are represented by shaded and $\alpha$-data by unshaded histograms. From [11].

\section{COUPP}

The Chicagoland Observatory for Underground Particle Physics (COUPP) [18] uses a similar technique of WIMP detection as PICASSO but instead of droplets dispersed in a gel matrix in modules, all the active bulk liquid is in one vessel, which then operates as a bubble chamber. Located in SNOLAB again, COUPP- 4 consisted of a 3 liter synthetic fused silica bell jar of $150 \mathrm{~mm}$ diameter sealed to a flexible stainless steel bellows and immersed in propylene glycol, serving as the hydraulic fluid, within a stainless steel pressure vessel. The flexible bellows ensured that the pressure inside and outside of the bell jar were the same, reducing the stress in the silica vessel. The bell jar was filled with $4 \mathrm{~kg}$ of superheated $\mathrm{CF}_{3} \mathrm{I}$ and topped with water to isolate the active liquid from contact with any surface apart from the smooth silica and water. Bubble nucleations were recorded three ways; a fast pressure transducer monitored the pressure rise in the chamber to track bubble growth, four piezoelectric transducers recorded the acoustic emissions, and two CCD cameras were used to photograph the chamber with a $20^{\circ}$ stereo angle at a frame rate of 100 per second. Images from these cameras were used to reconstruct the spatial coordinates of each bubble within the chamber. This was not possible in PICASSO due to a presence of the cloudy translucent gel.

A standard operating cycle of COUPP-4 began with the expansion of the $\mathrm{CF}_{3} \mathrm{I}$ from its normal state to the superheated state by reducing the pressure from 215 psia to the operating pressure of 30.1 psia over a period of five seconds. Following a 30 second period for pressure stabilization, the bubble chamber was live for the accumulation of dark matter data. The primary trigger for the experiment were frame-to-frame differences in the camera images, which initiated compression and capture of event data. The compression duration was 30 seconds, during which the data from the cycle were logged and the chamber was prepared for the next expansion. The average live-time fraction ranged from $78.8 \%$ to $82.2 \%$ depending on the operating temperature of the chamber, which determined the bubble nucleation threshold similarly to PICASSO. The $\mathrm{CF}_{3} \mathrm{I}$ was selected as the active liquid to give COUPP sensitivity in both the spin-dependent and spin-independent sectors thanks to the content of fluorine and iodine target nuclei respectively. Details of the COUPP-4 experiment, data reduction 

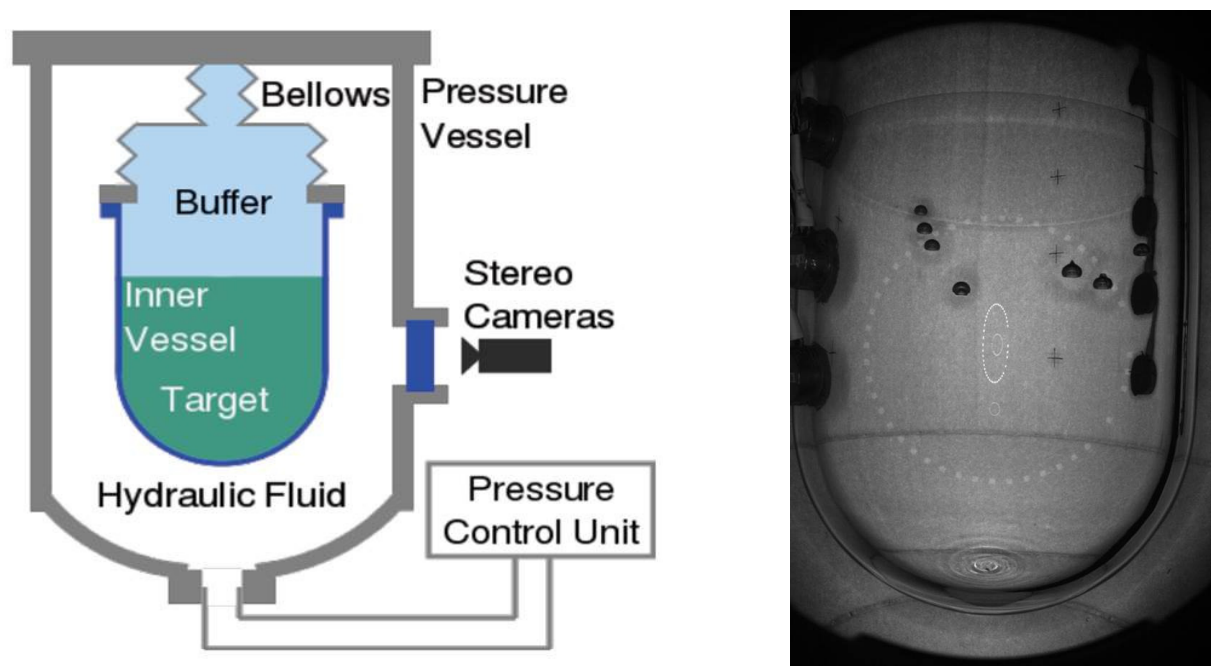

Figure 4: Left: Schematics of the COUPP bubble chamber showing the inner quartz vessel with the active liquid. Right: Image of a bubble in COUPP- 60 taken by one of the two CCD cameras used for triggering and spatial reconstruction.

and results of the operation in SNOLAB from September 2010 to August 2011 can be found in [13]. Between 2013 and 2014, the COUPP-60 filled with $60 \mathrm{~kg}$ of $\mathrm{CF}_{3} \mathrm{I}$ was running in SNOLAB and a publication with the first results is currently in preparation.

\section{PICO}

The PICO collaboration was formed in 2013 from the merger of PICASSO and COUPP groups to use the best of both experiments and build together the next generation of the superheated liquid detector. The droplet detectors are difficult to scale to larger masses, therefore the PICO detector uses the bubble chamber technique known from COUPP but instead of the $\mathrm{CF}_{3} \mathrm{I}$, which has an extremely poor efficiency for bubble nucleation from fluorine, limiting the sensitivity in spin-dependent sector, PICO uses $\mathrm{C}_{3} \mathrm{~F}_{8}$ as the active target. This new liquid has better spin-dependent sensitivity due to higher fluorine concentration, higher efficiency for detecting fluorine recoils and lower threshold without gamma and electron background. The collaboration combined operational principle of COUPP bubble chamber and fluid handling expertise from PICASSO to construct a prototype filled with $2.9 \mathrm{~kg}$ of $\mathrm{C}_{3} \mathrm{~F}_{8}$ and called PICO-2L. The detector uses a modified synthetic fused silica bell jar from COUPP4, together with 2 cameras, 3 acoustic transducers and a pressure sensor. All the technical details together with the data from the operation in SNOLAB from October 2013 to May 2014 are reported in a currently submitted publication [19].

The results from detectors PICASSO, COUPP and PICO show that experiments based on the superheated liquid technique are excellent for direct dark matter detection of both spin-dependent and spin-independent WIMP interactions, especially in the region of low WIMP masses. Thanks to their insensitivity to minimum ionizing backgrounds, ability to discriminate alpha-decay events from nuclear recoils and their low price, bubble chambers are ideal for scaling up. The next goal of the collaboration is to build PICO-250L, a superheated liquid member of the new generation of 


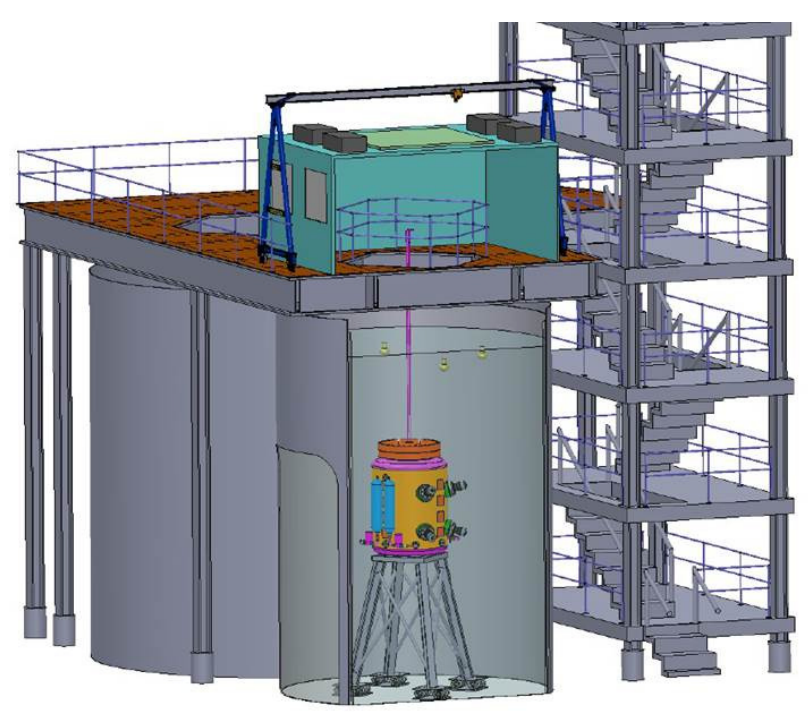

Figure 5: An illustration of PICO-250L with a visible pressure chamber submerged in the water bath.

ton scale dark matter detectors. Filled with $250 \mathrm{~kg}$ of $\mathrm{C}_{3} \mathrm{~F}_{8}$ (though the active liquid can be changed anytime), PICO-250L has the potential to be the leading experiment in the spin-dependent sector (as well as spin-independent sector in case of low-mass WIMPs) of direct dark matter searches as it has been the case with PICASSO and COUPP. Their published and expected 90\% C.L. limit plots for spin-dependent and spin-independent cross-sections are presented in Fig. 6.
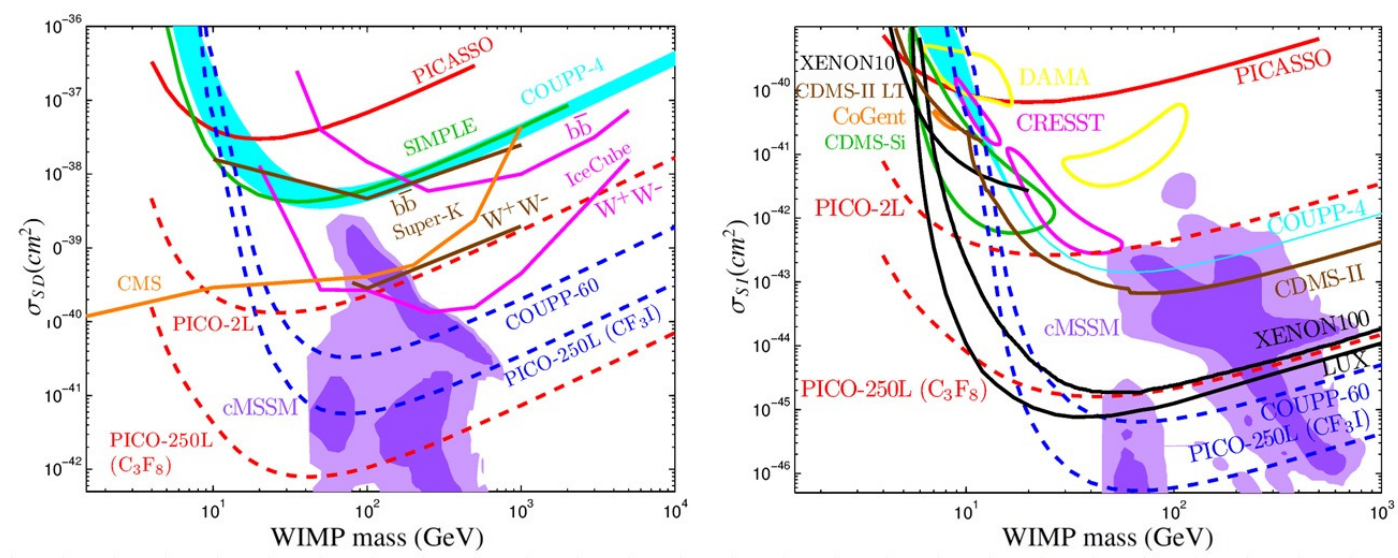

Figure 6: The previously reported limits (full lines) as well as theoretical models (dashed lines) of PICASSO, COUPP and PICO limits on spin-dependent (left) and spin-independent (right) WIMP elastic scattering. The real measurements from PICASSO, COUPP- 60 and PICO-2L will be published in near future. 
The PICO Collaboration would like to thank SNOLAB and its staff for providing an exceptional underground laboratory space and invaluable technical support. This material is based upon work supported by the U.S. Department of Energy, Office of Science, Office of High Energy Physics under award DE-SC-0012161. Fermi National Accelerator Laboratory is operated by Fermi Research Alliance, LLC under Contract No. De-AC02-07CH11359. Part of the research described in this paper was conducted under the Ultra Sensitive Nuclear Measurements Initiative at Pacific Northwest National Laboratory, a multiprogram national laboratory operated by Battelle for the U.S. Department of Energy. We acknowledge the National Science Foundation for their support including grants PHY1242637, PHY-0919526, and PHY-1205987. We acknowledge the support of the National Sciences and Engineering Research Council of Canada (NSERC) and the Canada Foundation for Innovation (CFI). We also acknowledge support from the Kavli Institute for Cosmological Physics at the University of Chicago. We acknowledge the financial support of the Spanish Ministerio de Economía y Competitividad, Consolider MultiDark CSD2009-00064 Grant. We acknowledge support from the Department of Atomic Energy (DAE), Govt. of India, under the project CAPP at SINP, Kolkata. We acknowledge the Czech Ministry of Education, Youth and Sports within the project LM2011027. We acknowledge technical assistance from Fermilab's Computing, Particle Physics, and Accelerator Divisions, and from A. Behnke at IUSB.

\section{References}

[1] G. Jungman, M. Kamionkowski, \& K. Griest, Physics Reports 267, 195-373 (1996)

[2] D. A. Glaser, Physical Review 87, 665-665 (1952)

[3] V. Zacek, Il Nuovo Cimento A 107, 291-298 (1994)

[4] J. Ellis, R. A. Flores, Physics Letters B 263, 259-266 (1991)

[5] V. A. Bednyakov, H. V. Kladpor-Kleingrothaus, S. G. Kovalenko, Physics Revue D 55, 503-514 (1997)

[6] R. E. Apfel, Nuclear Instruments and Methods 162, 603-608 (1979)

[7] H. Ing, R. Noulty, T. McLean, Radiation Measurements 27, 1-11 (1997)

[8] M. Barnabé-Heider et al., Nuclear Instruments and Methods in Physics Research A 555, 184-204 (2005)

[9] S. Archambault et al., New Journal of Physics 13, 043006 (2011)

[10] S. Archambault et al., Physics Letters B, 682, 185-192 (2009)

[11] F. Aubin et al., New Journal of Physics 10, 103017 (2008)

[12] E. Behnke et al., Physical Review Letters 106, 021303 (2011)

[13] E. Behnke et al., Physical Review D 86, 052001 (2012)

[14] R. Bernabei et al., The European Physical Journal C 67, 39-49 (2010)

[15] C. E. Aalseth et al., Physical Review Letters 106, 131301 (2011)

[16] G. Angloher, et al., The European Physical Journal C 72, 1971 (2012)

[17] S. Archambault et al., Physics Letters B 711, 153-161 (2012)

[18] E. Behnke et al., Science 319, 933-936 (2008)

[19] C. Amole et al., submitted 